\title{
Topology and performance redesign of complex structures by large admissible perturbations
}

\author{
D. Suryatama and M.M. Bernitsas
}

\begin{abstract}
A methodology for topology redesign of complex structures by LargE Admissible Perturbations (LEAP) is developed. LEAP theory is extended to solve topology redesign problems using 8-node solid elements. The corresponding solution algorithm is developed as well. The redesign problem is defined as a two-state problem. State S1 has undesirable characteristics and/or performance not satisfying certain designer specifications. The unknown State S2 has the desired structural response and locally optimum topology. First, the general nonlinear perturbation equations relating specific response of States S1 and S2 are derived. Next, a LEAP algorithm is developed which solves successfully two-state problems for large structural changes (on the order of $100 \%-300 \%$ ) of State S2, without repetitive finite element analyses, based on the initial State S1 and the specifications for State S2. The solution algorithm is based on an incremental predictor-corrector method. The optimization problems formulated in both the predictor and corrector phases are solved using commercial nonlinear optimization solvers. Minimum change is used as the optimality criterion. The designer specifications are imposed as constraints on modal dynamic and/or static displacement. The static displacement general perturbation equation is improved by static mode compensation thus reducing errors significantly. The moduli of elasticity of solid elements are used as redesign variables. The LEAP and optimization solvers are implemented in code RESTRUCT (REdesign of STRUCTures) which postprocesses finite element analyses results of MSC-NASTRAN. Several topology redesign problems are solved successfully by code RESTRUCT to illustrate the methodology and study its accuracy. Performance changes on the order of $3300 \%$ with high accuracy are achieved with only $3-5$ intermediate finite element analyses (iterations) to arrest
\end{abstract}

Received August 24, 1998

Revised manuscript received November 10, 1999

D. Suryatama and M.M. Bernitsas

Department of Naval Architecture and Marine Engineering, University of Michigan, 2600 Draper, Ann Arbor, MI 481092145, USA

e-mail: dsurya@engin.umich.edu,

michaelb@engin.umich.edu the error. Numerical applications show significant topological differences for varying redesign constraints.

Key words nonlinear perturbations, performance redesign, topology optimization, structural redesign, topology redesign

\section{1}

\section{Background}

Inverse design of structures (also called redesign) can be defined as a two-state problem. In a two-state problem, two structural states or designs are involved: the known initial State S1 and the unknown objective State $\mathrm{S} 2$. State $\mathrm{S} 1$ has undesirable performance which does not satisfy certain designer specifications, while State S2 satisfies all specifications. The relation between the two States S1 and S2 is highly nonlinear. The LargE Admissible Perturbation (LEAP) theory was developed to formulate and solve redesign problems (Bernitsas and Kang 1991; Bernitsas and Tawekal 1991; Bernitsas and Rim 1994; Beyko and Bernitsas 1993).

The theory and corresponding solution algorithm have been developed at the University of Michigan since 1983 and have solved two-state problems without trial and error or repetitive finite element analyses (Bernitsas 1994). LEAP can solve large change redesign problems without resorting to sensitivity or linearization. LEAP has been used successfully to solve various practical problems in structural analysis and design such as model reduction (Kang et al. 1992), model correlation (calibration) (Bernitsas and Tawekal 1991), and reliability (Beyko and Bernitsas 1993) using static, dynamic, and/ or stress constraints. The changes between $\mathrm{S} 1$ and $\mathrm{S} 2$ can be as large as $100 \%-300 \%$, depending on the scale and characteristics of the finite element model. Code RESTRUCT (REdesign of STRUCTures) - consisting of more than 30000 FORTRAN commands -implements LEAP theory and automates the redesign algorithm and computations. RESTRUCT postprocesses data generated by MSC-NASTRAN and can perform a complete 
redesign process with only one finite element analysis run. Postprocessing of data produced by other finite element codes is possible.

In the past, LEAP and RESTRUCT have been developed to solve resizing problems in which only crosssectional properties of elements - not the basic finite element mesh - are allowed to change. In resizing cases, acceptable performance in terms of static deflection, modal dynamic response, forced response amplitude or stress can be achieved without changing the topology of the structure. This may not be adequate, however, for structural designs that can also require an optimum topology to achieve their structural functions, conform with the boundary conditions, and support all the external loads.

Accordingly in this work, LEAP theory is extended to solve structural topology redesign problems. First, the general perturbation equations are developed for 8-node solid finite elements. Minimum change criterion and nonlinear perturbation equations for static displacement and modal dynamic response constraints are included. Several numerical applications, including benchmarking problems, are presented to illustrate the accuracy of the methodology for changes on the order of $3300 \%$ with only 4-6 finite element runs (iterations).

\section{1}

\section{Literature review}

The problem of structural redesign by perturbation can be formulated as an optimization problem. For linear (small) perturbation analysis, sensitivity methods can be used. Haug et al. (1986) and Choi et al. (1983) have studied extensively sensitivity methods which are efficient when analytical expressions for gradients are available. Validity of linear perturbation and sensitivity methods, however, is limited to small structural changes between S1 and S2 (Stetson 1975; Stetson and Harrison 1981; Sandstrom and Anderson 1982). Nonlinear perturbation methods were developed allowing for large structural changes - on the order of $100 \%$ to $300 \%$ from the initial State S1 - by implementing predictor-corrector solution schemes (Bernitsas and Kang 1991; Bernitsas and Tawekal 1991; Bernitsas 1994; Bernitsas and Rim 1994; Beyko and Bernitsas 1993; Hoff and Bernitsas 1985; Kang et al. 1992; Kang and Bernitsas 1994; Bernitsas et al. 1994; Kim and Bernitsas 1990; Hoff and Bernitsas 1986).

Development of LEAP (LargE Admissible Perturbation) methods can be summarized as follows. Static and modal dynamic redesign were introduced by Hoff and Bernitsas $(1985,1986)$ and then integrated by Kim and Bernitsas (1990) for solving redesign problems satisfying both objectives simultaneously. Structural model correlation for an offshore tower redesign were investigated by Bernitsas and Tawekal (1991) and structural redundancy by Kang and Bernitsas (1994). More complicated finite elements such as stiffened or unstiffened plate and shell were introduced by Bernitsas and Rim (1994) and Alzahabi and Bernitsas (1992). Beyko and Bernitsas (1993) solved a stochastic problem by defining the failure state of a structure by the general perturbation equation. Kang and Bernitsas (1994) formulated perturbation equations for stress redesign. At this stage, large admissible perturbation methods implemented in code RESTRUCT have been applied successfully to solve various redesign problems using spring, truss, bar, beam, plate, shell elements as well as combinations of redesign constraints or objectives such as static deflection, modal dynamic, and stress.

A first step toward solving topology redesign problems has been presented by Bernitsas et al. (1994). Static and dynamic topology redesign problems were investigated to find the optimum topology configuration of a cantilever plate. Results are consistent with those published in the literature (Yang and Chuang 1992). Shape and topology optimization have been studied extensively (Haber et al. 1995; Lipton and Diaz 1995; Bendsøe and Kikuchi 1988; Bremicker et al. 1991; Bernitsas and Suryatama 1999). Bendsøe and Kikuchi (1988) and Bremicker et al. (1991) developed the method of homogenization for topology and shape optimization. The method formulated a homogenized elasticity tensor of microstructure level to model a unit cell with a rectangular hole. The dimensions and orientation angles of the holes are used as the design variables for the optimization problem to minimize the compliance of a structure subject to a volume constraint. Yang and Chuang (1992) used linear programming to solve topology optimization problems minimizing the compliance subject to a volume constraint. In place of homogenization, they used a relationship between density and modulus of elasticity developed from an empirical formula to force the material density to be either zero or one.

Haber et al. (1995) presented the variable-topology shape optimization of elastic structures in $2 \mathrm{D}$ using the perimeter method. This imposes an upper bound constraint on the perimeter of the solid region to get a smooth design shape and to formulate a well-posed optimization problem for which solutions, comprised of solid and void materials, always exist. Hence, they minimized the compliance of a structure subject to an upper bound constraint on the volume and geometric constraints on the perimeter of the solid region.

The minimum compliance optimality criterion is also used by Lipton and Diaz (1995) to find the optimum layout of structures in $3 \mathrm{D}$. In this case, the optimization problem is decomposed into local and global optimization problems. In the local optimization level, the strain energy density of the structure is maximized, subject to a parameterization of orthotropic stiffness tensors of laminar microstructures in a prescribed volume fraction. In the global level, the minimum compliance is minimized subject to the material density within a fixed microstructure. 
In general, shape/topology optimization problems are solved using repeated finite element analysis runs. In this paper, finite element analysis (FEA) is performed only once for changes on the order of $100 \%-300 \%$. Intermediate FEA's may be needed to control the error in the redesign process for higher performance changes. Also, structural performance constraints such as static deflections and natural frequencies are used to obtain the desired structural response instead for just using volume or material density as constraints. Thus, in the process of optimizing topology, the LEAP algorithm produces a redesign which satisfies performance specifications.

\section{2}

\section{Structural perturbation equations}

The general perturbation equations express the performance of the unknown State S2 in terms of properties of $\mathrm{S} 1$ and redesign variables. Those equations for static displacement and frequency constraints are derived in this section. Unprimed and primed symbols refer to States S1 and S2, respectively. Information for mathematical symbols on this paper can be found in the nomenclature.

In the initial State S1, the static and modal dynamic equilibrium equations for finite element analysis are

$\mathbf{k u}=\mathbf{f}$,

$\left(\mathbf{k}-\omega^{2} \mathbf{m}\right) \boldsymbol{\Phi}=\mathbf{0}$.

For State S2, the counterpart equilibrium equations are

$$
\begin{aligned}
& \mathbf{k}^{\prime} \mathbf{u}^{\prime}=\mathbf{f}^{\prime}, \\
& \left(\mathbf{k}^{\prime}-\omega^{\prime 2} \mathbf{m}^{\prime}\right) \Phi^{\prime}=\mathbf{0} .
\end{aligned}
$$

States S1 and S2 quantities are related as follows: stiffness matrix:

$\mathbf{k}^{\prime}=\mathbf{k}+\Delta \mathbf{k}$

mass matrix:

$\mathbf{m}^{\prime}=\mathbf{m}+\Delta \mathbf{m}$,

mode shape:

$\boldsymbol{\Phi}^{\prime}=\boldsymbol{\Phi}+\Delta \boldsymbol{\Phi}$,

displacement:

$\mathbf{u}^{\prime}=\mathbf{u}+\Delta \mathbf{u}$,

where $\Delta$ represents change between the initial State S1 and the unknown State S2. Such changes obviously depend on the redesign variables. Substituting (5)-(8) into (3) and (4), we obtain
$\Delta \mathbf{u}=-\mathbf{u}+(\mathbf{k}+\Delta \mathbf{k})^{-1} \mathbf{f}^{\prime}$,

$\boldsymbol{\Phi}^{\prime T}\left(\Delta \mathbf{k}-\omega^{\prime 2} \Delta \mathbf{m}^{\prime}\right) \boldsymbol{\Phi}^{\prime}=-\boldsymbol{\Phi}^{\prime T}\left(\mathbf{k}-\omega^{\prime 2} \mathbf{m}^{\prime}\right) \boldsymbol{\Phi}^{\prime}$.

Here $\Delta \mathbf{k}$ and $\Delta \mathbf{m}$ are functions of redesign variables (identified by the designer) and can be computed based on S2 performance specified by the designer, such as, a few components of $\mathbf{u}^{\prime}, \boldsymbol{\omega}^{\prime}$, and/or $\boldsymbol{\Phi}^{\prime}$, as well as the properties of the initial State S1.

Let us assume that there are $p$ elements or groups of elements which the designer allows to change in the structure. Now, define $\alpha_{e}$ as a fractional change of a property of element group in State S1. In a single element or group, $\alpha_{e}$ may represent one property change such as torsion, bending, or stretching. Then, (5)-(6) can be expressed as

$$
\begin{aligned}
& \Delta \mathbf{k}=\sum_{e=1}^{p} \Delta \mathbf{k}_{e}=\sum_{e=1}^{p} \mathbf{k}_{e} \alpha_{e}, \\
& \Delta \mathbf{m}=\sum_{e=1}^{p} \Delta \mathbf{m}_{e}=\sum_{e=1}^{p} \mathbf{m}_{e} \alpha_{e},
\end{aligned}
$$

in case of linear dependence of $\mathbf{k}_{e}$ on $\alpha_{e}$. For instance, linear is the dependence of the beam element stiffness $\mathbf{k}_{e}$ on the cross-section moment of inertia $I$; and of the element mass $\mathbf{m}_{e}$ on the cross-section area $A$ (Kang and Bernitsas 1994). On the contrary, the plate element dependence of $\mathbf{k}_{e}$ on the plate thickness is cubic (Bernitsas and $\operatorname{Rim} 1994)$.

\section{1 Modal dynamic perturbation equations}

By substituting (5)-(8) and (11)-(12) into (10) (see Appendix A), the dynamic perturbation equations can be derived in terms of design variables $\alpha_{e}$ 's as

$\sum_{e=1}^{p}\left(\boldsymbol{\Phi}_{j}^{\prime T} \mathbf{k}_{e} \boldsymbol{\Phi}_{i}^{\prime}-\omega_{i}^{\prime 2} \boldsymbol{\Phi}_{j}^{\prime T} \mathbf{m}_{e} \boldsymbol{\Phi}_{i}^{\prime}\right) \alpha_{e}=$

$\omega_{i}^{\prime 2} \boldsymbol{\Phi}_{j}^{\prime T} \mathbf{m} \boldsymbol{\Phi}_{i}^{\prime}-\boldsymbol{\Phi}_{j}^{\prime T} \mathbf{k} \boldsymbol{\Phi}_{i}^{\prime}, \quad i, j=1,2, \ldots, n$,

where $n$ is the number of degrees of freedom of the finite element model in State S1.

In the objective State $\mathrm{S} 2$, the $n$ modal dynamic equations (13) can be separated into two categories. Diagonal, that is $i=j$ terms, which represent the Rayleigh quotient for mode $i$

$\sum_{e=1}^{p}\left(\boldsymbol{\Phi}_{i}^{\prime T} \mathbf{k}_{e} \boldsymbol{\Phi}_{i}^{\prime}-\omega_{i}^{\prime 2} \boldsymbol{\Phi}_{i}^{\prime T} \mathbf{m}_{e} \boldsymbol{\Phi}_{i}^{\prime}\right) \alpha_{e}=$

$\omega_{i}^{\prime 2} \boldsymbol{\Phi}_{i}^{\prime T} \mathbf{m} \boldsymbol{\Phi}_{i}^{\prime}-\boldsymbol{\Phi}_{i}^{\prime T} \mathbf{k} \boldsymbol{\Phi}_{i}^{\prime}$, 
and the off-diagonal terms, that is $i \neq j$ terms. These represent the orthogonality conditions in (13). Theoretically, orthogonality of modes with respect to one of $\mathbf{k}^{\prime}$ or $\mathbf{m}^{\prime}$ implies orthogonality with respect to the other. Numerically however, both conditions must be forced if $\boldsymbol{\Phi}_{j}^{\prime}$, for $j=1,2, \ldots, n$ are to represent modes of a real structure. These orthogonality conditions can be written as

$\sum_{e=1}^{p} \boldsymbol{\Phi}_{j}^{\prime T} \mathbf{k}_{e} \boldsymbol{\Phi}_{i}^{\prime} \alpha_{e}=-\boldsymbol{\Phi}_{j}^{\prime T} \mathbf{k} \boldsymbol{\Phi}_{i}^{\prime}$

$\sum_{e=1}^{p} \boldsymbol{\Phi}_{j}^{\prime T} \mathbf{m}_{e} \boldsymbol{\Phi}_{i}^{\prime} \alpha_{e}=-\boldsymbol{\Phi}_{j}^{\prime T} \mathbf{m} \boldsymbol{\Phi}_{i}^{\prime}$

for $i=1,2, \ldots, n$, and $j=i+1, i+2, \ldots, n$. Equations (14), (15), and (16) are the general perturbation equations for modal dynamics.

\section{2}

\section{Static perturbation equations}

Substituting (11) into (9), we derive the static general perturbation equation in terms of $\alpha_{e}$ 's.

$\Delta \mathbf{u}=\left(\mathbf{k}+\sum_{e=1}^{p} \mathbf{k}_{e} \alpha_{e}\right)^{-1} \mathbf{f}-\mathbf{u}$.

The assumption was made that $\mathbf{f}^{\prime}=\mathbf{f}$. If this is not the case, as in hydrodynamic loading on offshore platforms, an iteration is required within each increment to update the load. This case was studied by Tawekal and Bernitsas (1992). Expanding (17) and applying the linear relation between $\mathbf{u}^{\prime}$ and $\mathbf{f}^{\prime}$ introduced by Kim and Bernitsas (1990) (see Appendix B), we obtain the desired nodal point displacement of the $i$-th d.o.f. with respect to the $n_{r}$ extracted modes as a function of the redesign variables $\alpha_{e}$ 's

$u_{i}^{\prime}=\sum_{m=1}^{n_{r}} \frac{\Psi_{i m}^{\prime} A_{m}}{B_{m}+\sum_{e=1}^{p} C_{m e} \alpha_{e}}$,

where

$A_{m}=\sum_{j=1}^{n_{r}} \Psi_{j m}^{\prime} f_{j}, \quad B_{m}=\boldsymbol{\Phi}_{m}^{\prime T} \mathbf{k}_{e} \boldsymbol{\Phi}_{m}^{\prime}$

$C_{m e}=\boldsymbol{\Phi}_{m}^{\prime T} \mathbf{k}_{e} \boldsymbol{\Phi}_{m}^{\prime}$,

and $\Psi_{j m}^{\prime}$ represents the amplitude of $j$-th d.o.f. of mode $m$.

Fully nonlinear terms, as defined in (18), express the desired displacement $u_{i}^{\prime}$ in terms of the redesign variables $\alpha_{e}$. This approach gives more accurate results than previous static displacement equations which linearized the right-hand side of (18) using binomial expansion (Kim and Bernitsas 1990).
A modified static perturbation equation has been developed using static mode shape compensation (Bernitsas and Suryatama 1999). The new relation includes the static deflection shape as the zero-th mode in the modal expansion of nondimensional properties and static deflection in particular. The modified static perturbation equation improves the accuracy of the static redesign goals significantly and results are presented also in this paper. The new relations can be written as follows:

$u_{i}^{\prime}=u_{b i}-\sum_{m-1}^{n_{r}} \frac{\Psi_{i m}^{\prime} \sum_{e=1}^{p} \boldsymbol{\Phi}_{m}^{\prime T} \mathbf{k}_{e} \mathbf{u}_{b} \alpha_{e}}{\boldsymbol{\Phi}_{m}^{\prime T} \mathbf{k} \boldsymbol{\Phi}_{m}^{\prime}+\sum_{e=1}^{p} \boldsymbol{\Phi}_{m}^{\prime}{ }_{m} \mathbf{k}_{e} \boldsymbol{\Phi}_{m}^{\prime} \alpha_{e}}$,

which is the general perturbation equation for static deflection taking into account the static mode of the initial structure.

\section{3}

\section{LargE Admissible Perturbation (LEAP) algorithm}

Redesign by LargE Admissible Perturbation theory can be viewed as a two-step process. In the first step, the redesign problem is formulated as a two-state problem. In the first step, called Perturbation Approach to Redesign (PAR), the relationships between the response of the initial State S1 and the objective State S2 are derived. In the second step, a LEAP algorithm is developed to solve the implicit general perturbation equations derived in the first step. Thus, the values of the redesign variables that specify the objective design are obtained.

Since these general perturbation equations, e.g. the static and dynamic perturbation equations (14) and (18), are implicit nonlinear equations with respect to $\alpha_{e}$, they can not be solved directly. An incremental method, consisting of a predictor and a corrector phase in each increment, has been developed to solve these equations (Bernitsas and Kang 1991; Bernitsas and Tawekal 1991; Bernitsas 1994; Bernitsas and Rim 1994; Beyko and Bernitsas 1993; Hoff and Bernitsas 1985; Kang et al. 1992; Kang and Bernitsas 1994; Bernitsas et al. 1994; Kim and Bernitsas 1990; Hoff and Bernitsas 1986). In the predictor phase, the redesign variables $\alpha_{e}$ 's are predicted from (14) and/or (18). In the corrector phase, the objective eigenvectors $\boldsymbol{\Phi}_{i}^{\prime}$ are calculated. The redesign variables from the predictor phase are corrected using the predicted modes $\boldsymbol{\Phi}_{i}^{\prime}$ to satisfy (14)-(16) and (18).

The objective goal is achieved by incrementally changing the design of the initial structure until the objective state is achieved. In each increment, specified perturbations, on the order of $7 \%-30 \%$, are made until the objective response targets are achieved. At each increment, in both the predictor and corrector phases, the problem is formulated as an optimization problem and is solved using optimization solvers (Gill et al. 1983a,b; Tits and Zhou 1993). 
3

\section{Topology redesign}

3.1

\section{Formulation of topology redesign problem using solid elements}

In topology redesign, the initial State $\mathrm{S} 1$ is a solid continuum. Accordingly, it is modeled in finite element analysis by solid elements. 8-node solid elements, designated in MSC-NASTRAN as CHEXA elements, are utilized for our finite element models. The density and the elastic modulus of each element are used as redesign variables. The two variables are not related theoretically in the finite element method but a relation for porous materials is used in the next section.

In terms of the density variables, the consistent mass matrix of an isotropic element $\mathbf{m}_{e}$ can be written as

$\mathbf{m}_{e}=\varrho_{e} \int_{V} \mathbf{N}_{e}^{T} \mathbf{N}_{e} \mathrm{~d} V_{e}$

where $\varrho_{e}$ is the element density, $V_{e}$ is the element volume, and $\mathbf{N}_{e}$ represents the interpolation function matrix for each element. The relation between the $\alpha_{e}$ fractional changes of an element or a group of elements and its element mass $\mathbf{m}_{e}$ was provided in (12). For the entire structure that is composed of $n_{e}$ elements, the mass matrix is

$\mathbf{m}=\sum_{e=1}^{n_{e}} \varrho_{e} \mathbf{V}_{e}$

which shows that structural mass change can be achieved by changing the element density only.

Similarly, the stiffness equation $\mathbf{k}_{e}$ of an isotropic solid element is

$\mathbf{k}_{e}=E_{e} \int_{V} \mathbf{B}_{e}^{T} \mathbf{D}_{e} \mathbf{B}_{e} \mathrm{~d} V_{e}$,

where $E_{e}$ is the elastic modulus, $\mathbf{D}_{e}$ is the constitutive law matrix, and $\mathbf{B}_{e}$ is the strain-nodal displacement matrix. Since $E_{e}$ is constant for each element the stiffness of an element can be modified by changing $E_{e}$. The relation between the $\alpha_{e}$ fractional changes of an element or a group of elements and its element stiffness $\mathbf{k}_{e}$ is provided in (11).

\section{2}

\section{Optimization problem formulation}

In previous work done on structural redesign using the large admissible perturbation theory, minimum change has been used as the optimality criterion for computations of the optimal redesign. This function is selected to produce minimal structural change from State S1 to State $\mathrm{S} 2$ to achieve the designer's performance specifications. It gives satisfactory results not only for resizing/reshaping problems using truss, beam, and plate elements, but also for topology redesign using solid elements. This optimality criterion is

$\operatorname{minimize} \sum_{e=1}^{p} \alpha_{e}^{2}$.

The objective function will be subjected to one or more of the following equality/inequality constraints. There are $n_{\omega}$ natural frequency constraints, $n_{u}$ displacement constraints, $n_{a}$ admissibility constraints, and $2 p$ lower and upper bounds on redesign variables. The optimization problem can be formulated as

$\operatorname{minimize} f\left(\alpha_{e}\right)$,

subject to $n_{\omega}$ natural frequency constraints $(14), n_{u}$ displacement constraints (19), $n_{a}$ admissibility constraints (15)-(16) and $2 p$ lower and upper bounds on the redesign variables

$-1<\alpha_{e}^{-} \leq \alpha_{e} \leq \alpha_{e}^{+}, \quad e=1,2, \ldots, p$.

In each increment, the optimality criterion is given by (23). The criterion used in each increment, however, depends on the advance towards the optimum made to that increment. This is shown by Bernitsas and Kang (1991); Bernitsas and Tawekal (1991); Bernitsas (1994); Bernitsas and Rim (1994); Beyko and Bernitsas (1993); Hoff and Bernitsas (1985); Kang et al. (1992); Kang and Bernitsas (1994); Kim and Bernitsas (1990); Hoff and Bernitsas (1986); Alzahabi and Bernitsas (1992); Bernitsas and Suryatama (1999).

\section{3 \\ Algorithm for finding the optimum topology}

Following a complete iteration of redesign which includes a finite element run by MSC/NASTRAN and redesign by RESTRUCT, automated post-processing to define the new topology of a structure is carried out. The objective is to modify the input redesign data for RESTRUCT by freezing elements with low strain energy density. Since the external work is equal to the internal strain energy, by excluding elements which have low strain energy density in a structure, we only concentrate on modifying principal elements that carry the external load. Thus, stiffening only the principal elements leads to a gothic arc structure. The stiffened elements are identified as elements with high strain energy. A modified structural topology is based on these high strain energy density elements as shown in the numerical applications in Figs. 2-5. 
This process is repeated until redesign objectives are achieved and a satisfactory topology is obtained. For changes on the order of $3300 \%$ in structural performance the developed methodology requires only 4-6 FEA's (iterations). The following steps summarize this process.

Step 1. Perform a finite element analysis by MSC-NASTRAN; generate the RESTRUCT database for redesign.

Step 2. Redesign the structure by RESTRUCT to achieve the specified goals.

Step 3. Perform a finite element analysis by MSC-NASTRAN; generate the RESTRUCT database and strain energy density distribution on the structure.

Step 4. Does the new topology satisfy the specified structural performance goals? If yes, stop; otherwise go to Step 5.

Step 5. Modify RESTRUCT input data by eliminating redesign variables of elements that absorb strain energy density below a defined cut-off point (e.g. $6 \%-7 \%$ ) of the maximum element strain energy density. This step freezes elements from being redesigned in the next iteration. In subsequent iteration, frozen elements may be reactivated. Go to Step 2.

In one complete cycle from Step 1 to Step 5, the structure achieves the desired response specified by the designer as summarized in Tables 1-4 for the numerical applications in Section 4. Improved topologies of cantilever plate redesigns with minimum change objective are achieved in four to six topology redesign iterations as shown in Figs. 2-5.

\section{4}

\section{Numerical implementation}

The first step towards redesigning for topology changes is to implement solid elements in code RESTRUCT. A solid hexahedron with 8 nodes is selected. This element uses isoparametric shape functions to interpolate between the 8 nodal points using linear relations. Element sides can perform only translational movements (degrees of freedom), not rotations. The element is called CHEXA in MSC-NASTRAN and is considered more accurate for finite element analysis than a tetrahedron or a pentahedron element with the same type of nodal points (i.e. nodes at the element's apexes only).

The second step requires implementation of a nonlinear optimization solver at both the prediction and correction phases. Several optimization programs have been implemented in code RESTRUCT and have solved successfully many redesign applications. First, QPSOL (Gill et al. 1983a) was used when the general perturbation equations were linearized. Results from this optimizer are quite accurate for resizing problems and for approximately 40 redesign variables. Linearizations, however, contribute to inaccuracies for larger number of redesign variables. Thus, a nonlinear programming solver NPSOL (Gill et al. 1983b) or FSQP (Tits and Zhou 1993; Schittkowski 1986) is required to solve the problem with the nonlinear perturbation equations.

\section{1 \\ Structural redesign for resizing}

Results of various redesigns of a solid plate cantilever are presented in this section. The original structure is shown in Fig. 1 and is modeled using 160 solid elements. It is a cantilever plate structure with an inplane load of $300 \mathrm{~N}$ at the tip of the cantilever. Static, dynamic, and combination of static and dynamic redesigns are performed with the minimum change optimality criterion. The structure is redesigned to reduce or increase maximum vertical deflection of the loaded node which in State S1 is $0.0106 \mathrm{~mm}$. The structure is redesigned also to shift the second natural frequency of the vertical bending mode shape. A combination of these two objectives in redesign is used as well. Best results are achieved by using FSQP as the nonlinear optimization solver and implementing an improved LEAP algorithm for static redesign which includes static mode compensation. In order to put into perspective the accuracy of the developed algorithm, several examples are shown using NPSOL and QPSOL as well as the LEAP static redesign algorithm without static compensation. Results are shown in Tables 1-5. Cases e1-e4 of Table 5 implement the static compensation redesign algorithm using FSQP and show very high accuracies for $100 \%$ changes in redesign objectives with only a single finite element analysis. Redesigns for structural changes as well as for structural/topology changes are computed using the San Diego Cray-90 Supercomputer.

Table 1 shows results of redesigns using QPSOL or NPSOL. Cases a1 and a3 show that the nonlinear static prediction of equation (18) used by NPSOL gives smaller error than the linearized version of (18) used by QPSOL. The error of NPSOL results can still be reduced by selecting more extracted modes $n_{r}$ in the static perturbation equations as shown in cases a 6 and a7. In these two cases, 40 extracted modal dynamic modes are used compared to 20 used in cases a1-a5. The difference between cases a 6 and $a 7$ is that an active admissible cognate space is used in case a6. An active admissible cognate space is defined as the set of modes selected from the extracted modal dynamic modes to be used for solving the general perturbation equations on the basis of nontrivial admixture coefficients. Those coefficients define the mutual interaction between changes of modes (Bernitsas and Tawekal 1991).

Cases a2 and a5 show that dynamic redesign always produces accurate results, regardless of the optimization solver being used. This accuracy is due to the fact that the desired natural frequency of the dynamic perturbation (14) is linear with respect to the redesign variables $\alpha_{e}$ 's. 


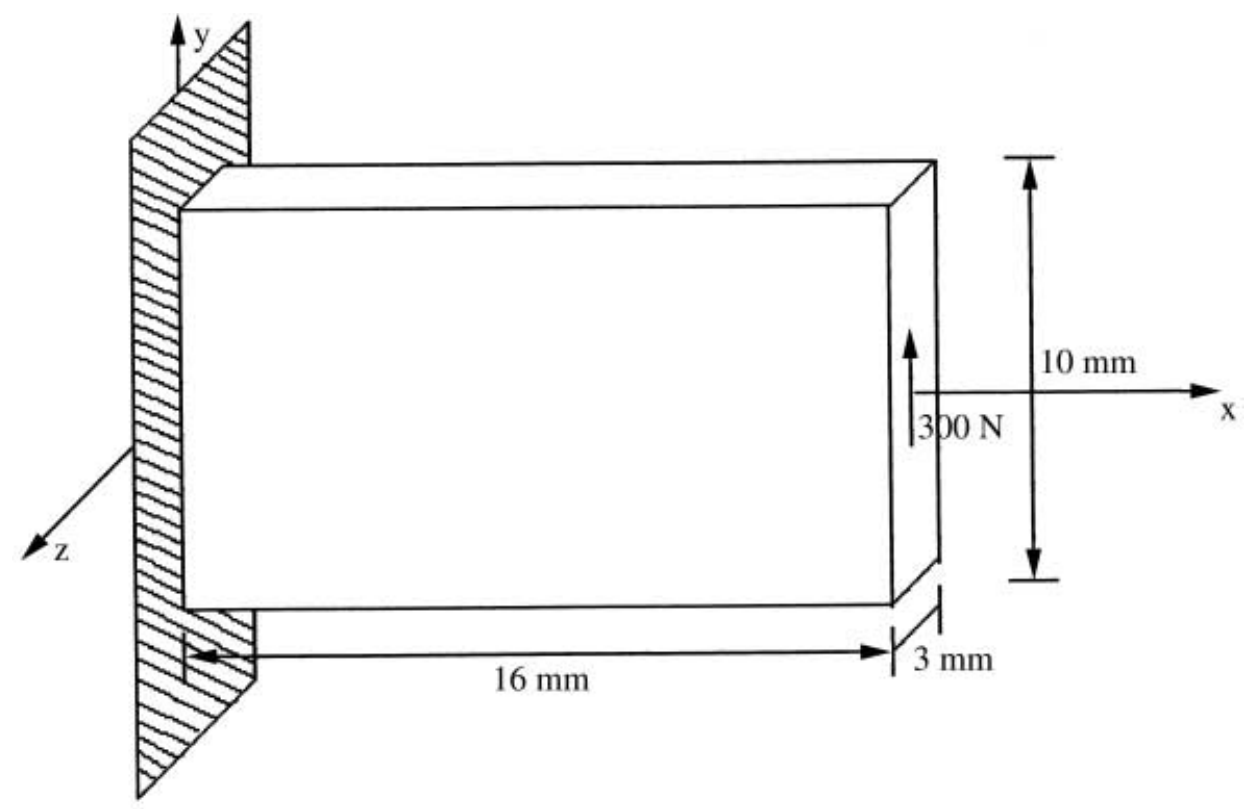

Fig. 1 Initial Structure S1: 3D cantilever plate. Properties: $E=2.07 \times 10^{5} \mathrm{Mpa} ; \varrho=7.833 \times 10^{-9} \mathrm{Nsec}^{2} / \mathrm{mm}^{4} ; \nu=0.3$. Response: $f_{1}=9702.04 \mathrm{~Hz}, f_{2}=26147.6 \mathrm{~Hz}, v_{289}=v_{102}=0.01 \mathrm{~mm}$. Elements: 160 solid hexa elements, 1122 d.o.f.'s

Table 1 Comparison between QPSOL and NPSOL results

\begin{tabular}{|c|c|c|c|c|c|c|c|c|c|c|}
\hline \multirow[t]{2}{*}{ Case } & \multicolumn{2}{|c|}{ Static redesign } & \multicolumn{2}{|c|}{ Dynamic redesign } & \multirow{2}{*}{$\begin{array}{l}\# \text { of } \\
\alpha_{e}\end{array}$} & \multirow[t]{2}{*}{$n_{r}$} & \multirow{2}{*}{$\begin{array}{l}\text { Incr. } \\
(\%)\end{array}$} & \multirow{2}{*}{$\begin{array}{l}\# \text { of } \\
\text { Incr. }\end{array}$} & \multirow[t]{2}{*}{ Solver } & \multirow{2}{*}{$\begin{array}{l}\mathrm{CPU}^{2} \\
(\mathrm{sec})\end{array}$} \\
\hline & $u_{289}^{\prime} / u_{289}$ & Error $(\%)^{1}$ & $\omega_{2}^{\prime 2} / \omega_{2}^{2}$ & Error $(\%)$ & & & & & & \\
\hline a1 & 0.755 & -16.556 & & & 80 & 20 & 7 & 4 & QPSOL & $\mathrm{N} / \mathrm{A}$ \\
\hline $\mathrm{a} 2$ & 0.755 & -16.566 & 1.500 & 1.400 & 80 & 20 & 7 & 6 & QPSOL & $\mathrm{N} / \mathrm{A}$ \\
\hline a3 & 0.755 & -11.126 & & & 80 & 20 & 7 & 4 & NPSOL & 148 \\
\hline $\mathrm{a} 4$ & 0.755 & -10.331 & & & 80 & 20 & 4 & 7 & NPSOL & 392 \\
\hline a5 & & & 1.500 & 0.000 & 80 & 20 & 4 & 11 & NPSOL & 468 \\
\hline a6 & 0.755 & -7.751 & & & 80 & 40 & 7 & 4 & NPSOL & 831 \\
\hline a7 & 0.755 & -7.577 & & & 80 & 40 & 7 & 4 & NPSOL & 787 \\
\hline
\end{tabular}

${ }^{1}$ No static compensation

${ }^{2}$ CPU time of CRAY-90 at San Diego Super Computer

In Table 2, results obtained by NPSOL and FSQP are compared. The two optimizers have comparable levels of accuracy as shown in cases b1 and b2. In case $\mathrm{b} 4$, the convergence tolerance for nonlinear equality constraints is set equal to $10^{-7}$. This provides more accurate results compared to case b3 with the convergence tolerance for nonlinear equality constraints set equal to $10^{-4}$. Results of cases b3 and b4 are satisfactory, even though more redesign variables are used. In cases b3 and b4, elastic moduli of each element are used as the redesign variables while in cases b1 and b2 only elastic moduli are used. Cases b1-b4 show that the accuracy of the general perturbation equation for static redesign objective is less than the equation for modal dynamic objective. Thus, improvements are required for the static general perturbation equation by using static mode compensation techniques (see Section 2.2).
Redesign with large structural changes on the order of $100 \%$ to $200 \%$ are solved successfully using RESTRUCT with the FSQP solver. In Table 3, case $\mathrm{c} 1$ shows a reduction of the maximum static deflection and case $\mathrm{c} 2$ shows the combination of a reduction of the static deflection and an increase in the second natural frequency. In all of these cases, the resulting errors are acceptable and in case $\mathrm{c} 5$ the error is negligible. These results show that RESTRUCT can solve highly nonlinear redesign problems for large structural changes. It should be noted, however, that some redesign problems where the structure deteriorates (large reduction stiffness) should be studied more carefully. In case $\mathrm{c} 3$, the maximum static deflection is increased and in case $c 4$ the second natural frequency is reduced. These problems result in higher errors and need further investigation to understand better cases where the extracted modes do not represent a high enough cumulative level of strain energy. 
Table 2 Redesign results of NPSOL and FSQP

\begin{tabular}{|c|c|c|c|c|c|c|c|c|c|c|}
\hline \multirow[t]{2}{*}{ Case } & \multicolumn{2}{|c|}{ Static redesign } & \multicolumn{2}{|c|}{ Dynamic redesign } & \multirow{2}{*}{$\begin{array}{l}\# \text { of } \\
\alpha_{e}\end{array}$} & \multirow[t]{2}{*}{$n_{r}$} & \multirow{2}{*}{$\begin{array}{l}\text { Incr. } \\
(\%)\end{array}$} & \multirow{2}{*}{$\begin{array}{l}\text { \# of } \\
\text { Incr. }\end{array}$} & \multirow[t]{2}{*}{ Solver } & \multirow{2}{*}{$\begin{array}{l}\mathrm{CPU}^{2} \\
(\mathrm{sec})\end{array}$} \\
\hline & $u_{289}^{\prime} / u_{289}$ & Error $(\%)^{1}$ & $\omega_{2}^{\prime 2} / \omega_{2}^{2}$ & Error $(\%)$ & & & & & & \\
\hline b1 & 0.934 & -6.278 & & & 80 & 40 & 7 & 1 & NPSOL & 70 \\
\hline $\mathrm{b} 2$ & 0.934 & -6.362 & & & 80 & 40 & 7 & 1 & FSQP & 51 \\
\hline b3 & 0.932 & -5.251 & 1.070 & 2.078 & 160 & 80 & 7 & 1 & FSQP & 229 \\
\hline $\mathrm{b} 4$ & 0.932 & -4.963 & 1.070 & 2.062 & 160 & 80 & 7 & 1 & FSQP & 319 \\
\hline
\end{tabular}

${ }^{1}$ No static compensation

${ }^{2}$ CPU time of CRAY-90 at San Diego Super Computer

Table 3 Large structural changes of the solid cantilever

\begin{tabular}{lllllllllll}
\hline Case & \multicolumn{2}{c}{$\begin{array}{c}\text { Static redesign } \\
\text { Error }(\%)^{1}\end{array}$} & $\begin{array}{c}\text { Dynamic redesign } \\
\omega_{2}^{\prime 2} / \omega_{2}^{2}\end{array}$ & Error $(\%)$ & $\begin{array}{l}\# \text { of } \\
\alpha_{e}\end{array}$ & $n_{r}$ & $\begin{array}{l}\text { Incr. } \\
(\%)\end{array}$ & $\begin{array}{l}\text { \# of } \\
\text { Incr. }\end{array}$ & $\begin{array}{c}\text { Solver } \\
u_{289}^{\prime} / u_{289}\end{array}$ & $\begin{array}{l}\text { CPU } \\
(\mathrm{sec})\end{array}$ \\
\hline \hline $\mathrm{c} 1$ & 0.500 & -6.050 & & & 80 & 80 & 15 & 15 & FSQP & 2762 \\
c2 & 0.500 & -6.549 & 2.000 & 7.608 & 160 & 80 & 25 & 4 & FSQP & 1094 \\
c3 & 1.7515 & -12.749 & & & 80 & 80 & 15 & 4 & FSQP & 752 \\
c4 & & 0.500 & 6.154 & 80 & 80 & 15 & 5 & FSQP & 664 \\
c5 & & 3.000 & 0.065 & 80 & 20 & 15 & 8 & FSQP & 100 \\
\hline
\end{tabular}

${ }^{1}$ No static compensation

${ }^{2}$ CPU time of CRAY-90 at San Diego Super Computer

Results for static redesign presented in the previous applications are derived based on a LEAP static perturbation algorithm implementing equation (18). The accuracy of that algorithm is less than the dynamic redesign algorithm. Static mode compensation techniques were used in developing equation (19) and the results presented in Tables $4-5$ show improved accuracy of the corresponding LEAP algorithm.

In case $\mathrm{d} 1$ of Table 4 , the plate cantilever is redesigned for a reduction of the maximum static deflection at the loaded node by a factor of two. This case is performed using 80 extracted dynamic modes of the free vibration analysis and it has a relatively low error of $-6.050 \%$ without any intermediate finite element analyses or static mode compensation. The same problem, solved using the newly developed algorithm as shown in case e 2 in Table 5 , produces a lower error of $2.7499 \%$ using only 20 extracted modes. Note that in this case the reduction of the extracted modes is very significant in terms of the computational time. Here the total CPU time of case 22 is reduced by a factor of 4 with respect to the $\mathrm{CPU}$ in case $\mathrm{d} 1$. Cases $\mathrm{e} 1$ and $\mathrm{e} 3$ are similar to case d1. Redesign e1 is performed using $7 \%$ fractional changes at each increment of the predictorcorrector algorithm and, thus, requires higher number of increments. Case e2 is performed with only 5 extracted dynamic modes and still has a slightly lower error than case $\mathrm{d} 1$.

In case $\mathrm{d} 2$ in Table 4 , concurrent static and dynamic objectives are imposed. In this case, the maximum static deflection is reduced by a factor of two at the loaded node and the second bending natural frequency at the vertical direction is increased by a factor of two. The accuracy, as shown in Table 4, is nearly equal to the static only redesign problem in case $\mathrm{d} 1$ with static redesign error of $-6.549 \%$ and dynamic redesign error of $7.608 \%$. The dynamic redesign error, in this case, is rather high as the general perturbation equation for natural frequency is very accurate. This error is due to the inaccuracies of the static perturbation equation (18) which affect the prediction of the correct natural frequency. Using newly developed LEAP algorithm based on perturbation equation (19) which includes static mode compensation, this problem is remedied and results in low errors for both the static and dynamic objectives as shown in case e 4 in Table 5.

\section{2}

\section{Topology redesign of the solid cantilever plate}

The solid cantilever plate shown in Fig. 1 is redesigned in this section for structural performance and topological changes. The structure is analyzed by two finite element models with 160 and 640 solid hexa elements. The two finite element models are used to redesign the benchmarking problem solved by Haber et al. (1995); Lipton and Diaz (1995); Bendsøe and Kikuchi (1988); Bremicker et al. (1991); Bernitsas and Suryatama (1999). The produced topologies are compared to the uniqueness of the solution algorithm. Topological evolution during the redesign process is discussed to understand how the struc- 
Table 4160 solid elements, 1122 d.o.f.'s, solid plate cantilever w/o using static mode compensation

\begin{tabular}{llllllllll}
\hline Case & \multicolumn{2}{c}{$\begin{array}{c}\text { Static redesign } \\
\text { (2) }\end{array}$} & \multicolumn{2}{c}{ Dynamic redesign } & \# of & \# of red. \\
increments & variables & $\begin{array}{l}\text { \# of } \\
\text { modes }\end{array}$ & $\begin{array}{l}\text { Incr. } \\
(\%)\end{array}$ & $\begin{array}{l}\mathrm{CPU}^{2} \\
(\mathrm{sec})\end{array}$ \\
\hline \hline $\mathrm{d} 1$ & 0.500 & -6.050 & & & 15 & 80 & 80 & 15 & 2762 \\
$\mathrm{~d} 2$ & 0.500 & -6.549 & 2.000 & 7.608 & 4 & 160 & 80 & 25 & 1094 \\
$\mathrm{~d} 3$ & & & 3.000 & 0.065 & 8 & 80 & 20 & 15 & 100 \\
\hline
\end{tabular}

${ }^{1}$ No static compensation

${ }^{2}$ CPU time of CRAY-90 at San Diego Super Computer

Table 5160 solid elements, 1122 d.o.f.'s, solid plate cantilever using static mode compensation

\begin{tabular}{|c|c|c|c|c|c|c|c|c|c|}
\hline \multirow[t]{2}{*}{ Case } & \multicolumn{2}{|c|}{ Static redesign } & \multicolumn{2}{|c|}{ Dynamic redesign } & \multirow{2}{*}{$\begin{array}{l}\# \text { of } \\
\text { increments }\end{array}$} & \multirow{2}{*}{$\begin{array}{l}\text { \# of red. } \\
\text { variables }\end{array}$} & \multirow{2}{*}{$\begin{array}{l}\# \text { of } \\
\text { modes }\end{array}$} & \multirow{2}{*}{$\begin{array}{l}\text { Incr. } \\
(\%)\end{array}$} & \multirow{2}{*}{$\begin{array}{l}\mathrm{CPU}^{2} \\
(\mathrm{sec})\end{array}$} \\
\hline & $u_{289}^{\prime} / u_{289}$ & Error $(\%)^{1}$ & $\omega_{2}^{\prime 2} / \omega_{2}^{2}$ & Error (\%) & & & & & \\
\hline e1 & 0.5000 & 2.6130 & & & 10 & 80 & 20 & 7 & 1465 \\
\hline $\mathrm{e} 2$ & 0.5000 & 2.7499 & & & 5 & 80 & 20 & 15 & 678 \\
\hline e3 & 0.5000 & -5.8858 & & & 5 & 80 & 5 & 15 & 420 \\
\hline $\mathrm{e} 4$ & 0.5000 & 2.5676 & 2.0000 & -0.9320 & 5 & 80 & 20 & 15 & 706 \\
\hline
\end{tabular}

${ }^{1}$ With static compensation

${ }^{2}$ CPU time of CRAY-90 at San Diego Super Computer

ture evolves from a solid plate cantilever to a gothic arc like structure. In both cases, the accuracy of the LEAP algorithm in predicting large structural changes on the order of $3300 \%$ with $4-6$ intermediate finite element analyses is also discussed.

The algorithm developed in this work based on LEAP theory and explained in Section 2.3 achieves both structural performance and topological objectives simultaneously. Each redesign case requires $4-6$ iterations and in each iteration the following steps are carried out: a finite element run, a redesign by RESTRUCT and a strain energy density post-processing. The iterations required by the LEAP algorithm are significantly fewer than those specified by Haber et al. (1995); Lipton and Diaz (1995); Bendsøe and Kikuchi (1988); Bremicker et al. (1991); Bernitsas and Suryatama (1999). In addition to these advantages, structural performance and topological objectives are achieved.

Redesigns for structural performance and topological objectives are studied below for static, modal dynamic, and simultaneous static and modal dynamic objectives. Results for redesign of the cantilever plate to reduce the maximum static displacement are listed in Tables 6 and 7 . The baseline structure, as shown in Fig. 1, has maximum deflection of $0.0106 \mathrm{~mm}$ at two nodes where the loads are applied. Both finite element models are subjected to large structural changes using the elastic modulus of all elements as redesign variables. The maximum displacement is reduced by a factor of 33 from $0.0106 \mathrm{~mm}$ to $0.000321 \mathrm{~mm}$ at the end of iteration 6 . In each iteration, large topological changes are achieved as shown in Figs. 2 and 3. A finite element run carried out after each iteration is required in order to modify the RESTRUCT input data. Thus, error is not allowed to propagate and elements with low strain energy density are not used as redesign variables in the next iteration. These intermediate finite element runs are not needed if only structural performance changes are being sought. The LEAP algorithm has been successfully applied to compute large structural changes with only one finite element run and satisfactory accuracy for static and/or modal dynamic objectives as shown in Section 4.1.

Topological changes at each iteration based on the strain energy density distribution are illustrated in Figs. 2 and 3 . In these figures, elements with low strain energy density are shown in blue or dark blue colors. Elements in other colors (red, yellow, and cyan) reflect high strain energy density elements. These elements become gradually stiffer in the redesign process. We can see also from Iteration 1 in Figs. 2 and 3 that the stiffening process starts at the nodes where the load is and at the corners of the clamped end. In the stiffening process, more high strain energy density elements are formed to connect the stiffened areas as shown in Iteration 2 in Figs. 2 and 3. Stiffening in the centre of the plate creates the cross bracing progressively until Iteration 6 in Figs. 2 and 3. It is worth noting that the produced structure does not have straight elements like a truss but curved like a Gothic arc structure.

Since Figs. 2 and 3 show the redesign process for the two different models with 160 and 640 elements, respectively. Within the resolution error, these two figures show the uniqueness of the redesign process. Iterations 2 and 3 of the two figures show that stiffening starts at the same area and progresses to the centre structural region. The slight difference of the cross bracing is due 

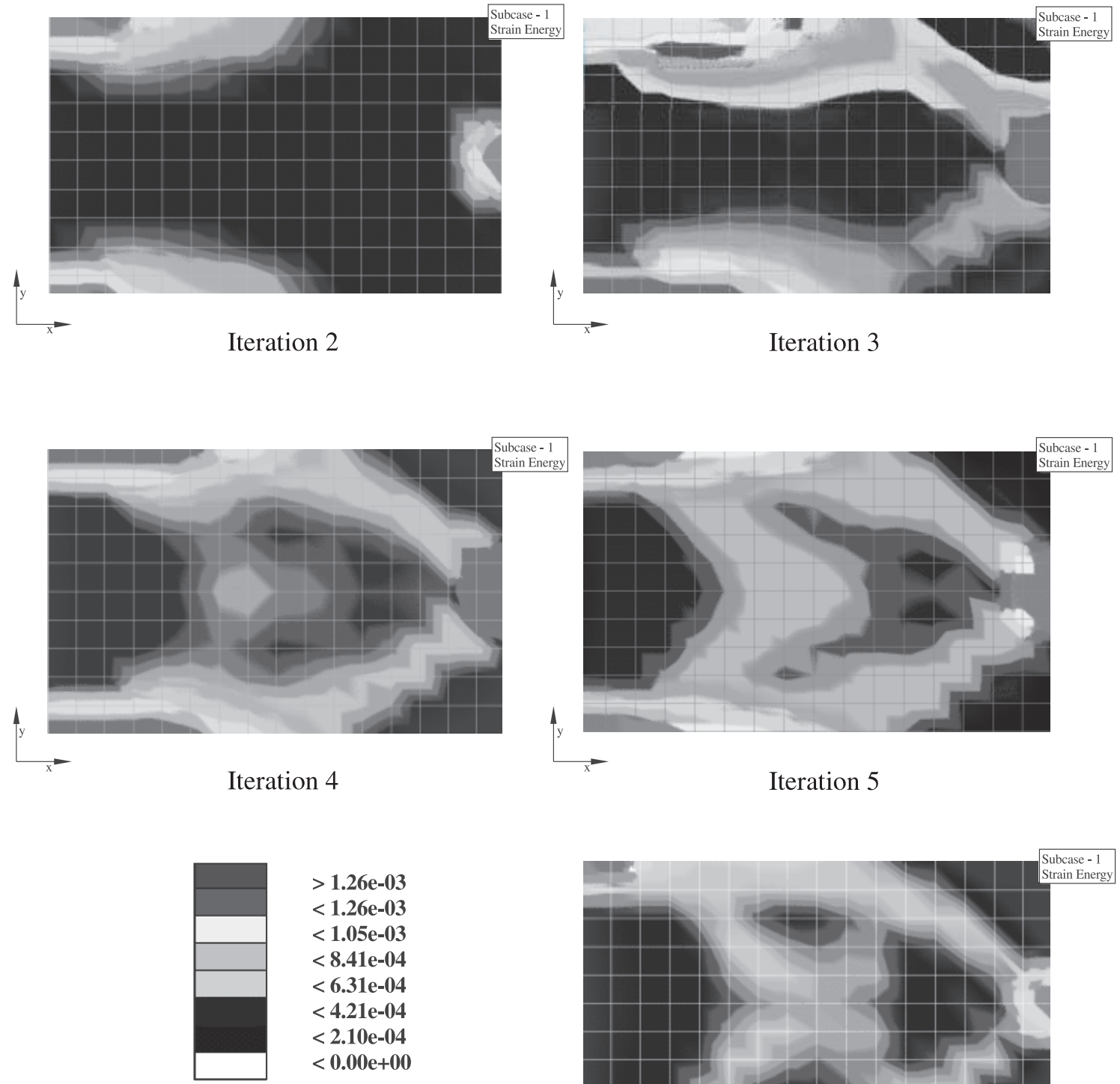

$\max =1.47 \mathrm{e}-03$

$\min =0.00 \mathrm{e}+00$

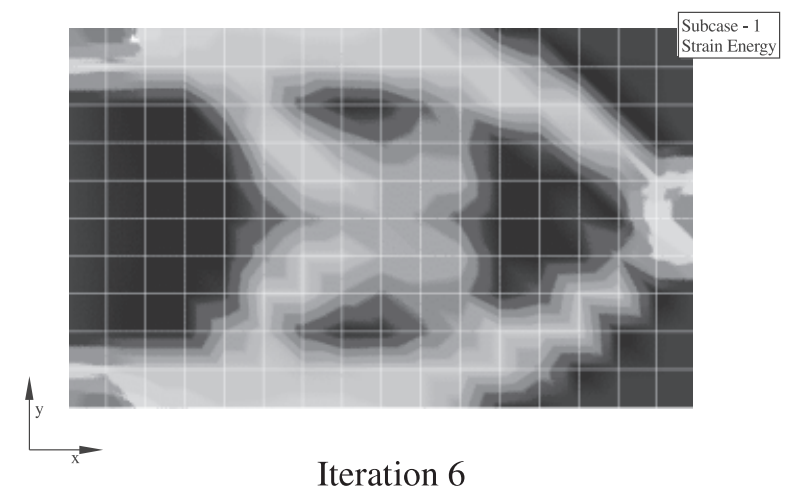

Fig. 2 Static strain energy after static redesign (160 solid element cantilever plate); $u_{102}^{\prime} / u_{102}=0.0303$

to chattering as more finite elements are used in Fig. 3. Topology and structural redesign for changing the second natural frequency is performed next. The structure shown in Fig. 1 is redesigned to increase its second natural frequency of $26147.6 \mathrm{~Hz}$. The redesign goals are shown in terms of the ratios of eigenvalues $\omega_{2}^{\prime 2} / \omega_{2}^{2}$ in Table 8 . Results are very accurate with errors of less than about $2.5 \%$ for $180 \%$ changes in the eigenvalue at each iteration. The total change in $\omega_{2}^{2}$ is $1089 \%$ of the baseline structural response. Topological changes are shown in Fig. 4.

Simultaneous static and modal dynamic redesigns are performed next. The solid plate cantilever is redesigned to reduce its maximum static displacement and at the same time increase its second natural fre- quency. The results of the structural performance changes are shown in Table 9 while the topological changes are shown in Fig. 5. Figure 5 shows the normalized combination of static and dynamic strain energy density distributions. The plots of Fig. 5 are constructed by normalizing each element strain energy density with respect to the maximum value in each analysis. Then, the normalized results from the static and modal dynamic analyses are added up to show the influence of both redesign objectives.

In Fig. 5, the plots of iterations 3 and 4 show the influence of modal dynamic and static redesign objectives. The left side of each plot - closer to the fixed endexhibits the significant features of the modal dynamic redesign in which cross bracing is formed similar to Fig. 4. 


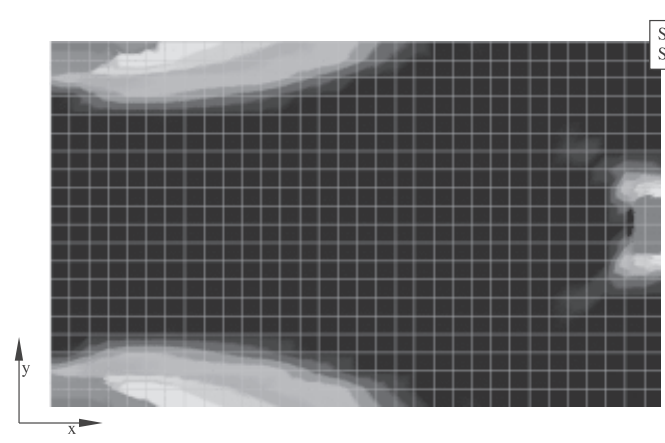

Iteration 2

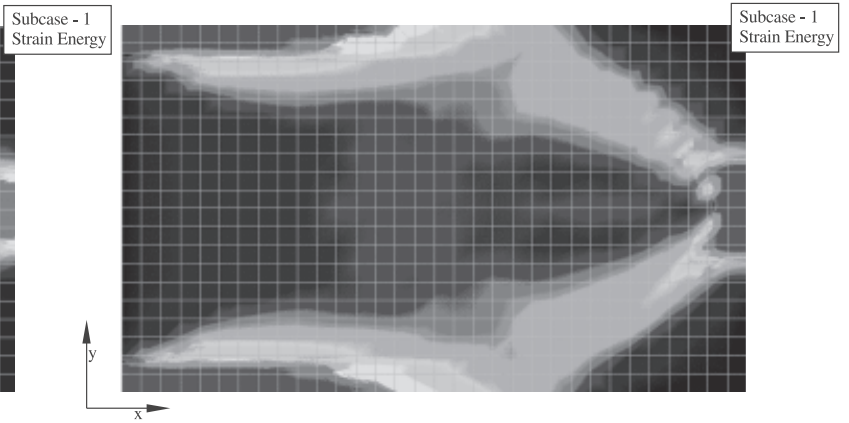

Iteration 3

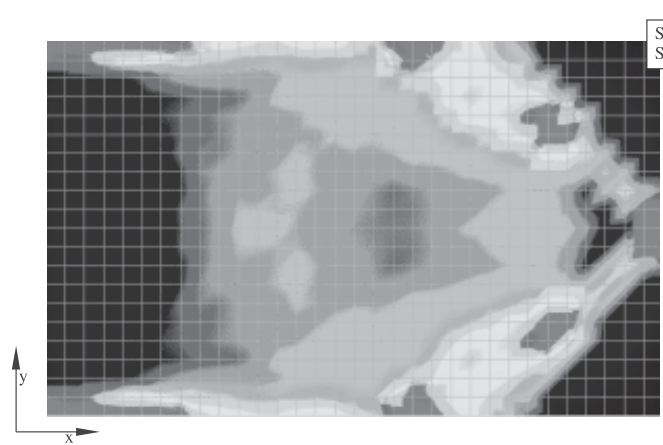

Iteration 4

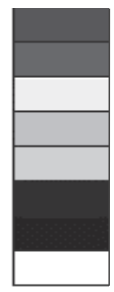

$>2.73 \mathrm{e}-04$

$<2.73 \mathrm{e}-04$

$<2.28 \mathrm{e}-04$

$<1.82 \mathrm{e}-04$

$<1.37 \mathrm{e}-04$

$<9.10 \mathrm{e}-05$

$<4.55 \mathrm{e}-05$

$<0.00 \mathrm{e}+00$

$\max =9.51 \mathrm{e}-04$

$\min =0.00 \mathrm{e}+00$

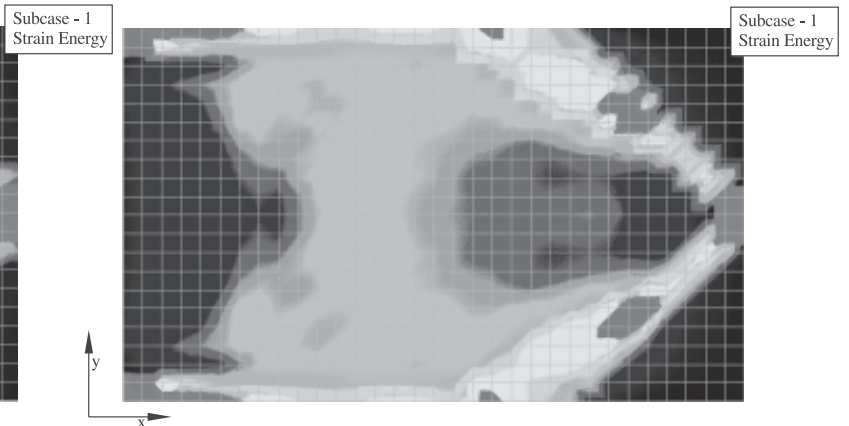

Iteration 5

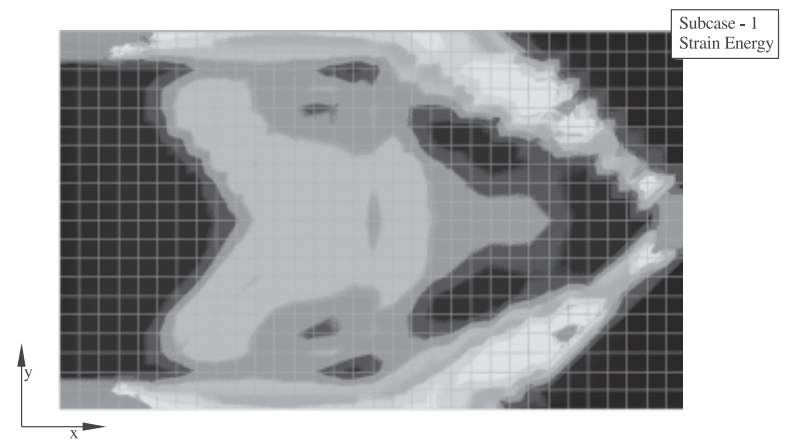

Iteration 6

Fig. 3 Static strain energy after static redesign ( 640 solid element cantilever plate); $u_{1137}^{\prime} / u_{1137}=0.0286$

Table 6 Results of the 160 element (1122 d.o.f.'s) model at each iteration for static redesign objective

\begin{tabular}{|c|c|c|c|c|c|c|}
\hline \multirow{2}{*}{$\begin{array}{l}\text { Iter } \\
\#\end{array}$} & \multicolumn{2}{|c|}{ Static redesign } & \multirow{2}{*}{$\begin{array}{l}\text { \# of } \\
\text { increments }\end{array}$} & \multirow{2}{*}{$\begin{array}{l}\text { \# of redesign } \\
\text { variables }\end{array}$} & \multirow{2}{*}{$\begin{array}{l}\text { \# of } \\
\text { modes }\end{array}$} & \multirow{2}{*}{$\begin{array}{l}\text { Increment } \\
\text { size }(\%)\end{array}$} \\
\hline & $u_{289}^{\prime} / u_{289}$ & Error $(\%)^{*}$ & & & & \\
\hline 1 & 0.937 & -0.402 & 1 & 80 & 10 & 15 \\
\hline 2 & 0.523 & 2.456 & 4 & 58 & 30 & 15 \\
\hline 3 & 0.523 & 6.043 & 4 & 62 & 30 & 15 \\
\hline 4 & 0.523 & 6.419 & 4 & 50 & 30 & 15 \\
\hline 5 & 0.523 & 5.171 & 4 & 47 & 80 & 15 \\
\hline 6 & 0.523 & 0.358 & 1 & 30 & 80 & 15 \\
\hline
\end{tabular}

* Error is not cumulative since it is arrested by each FEA 
Table 7 Results of the 640 element (4158 d.o.f.'s) model at each iteration for static redesign objective

\begin{tabular}{lllllll}
\hline $\begin{array}{l}\text { Iter } \\
\#\end{array}$ & \multicolumn{2}{c}{$\begin{array}{c}\text { Static redesign } \\
u_{1137} / u_{1137}\end{array}$} & $\begin{array}{l}\text { Error }(\%)^{*} \\
\text { increments }\end{array}$ & $\begin{array}{l}\text { \# of redesign } \\
\text { variables }\end{array}$ & $\begin{array}{l}\text { \# of } \\
\text { modes }\end{array}$ & $\begin{array}{l}\text { Increment } \\
\text { size }(\%)\end{array}$ \\
\hline \hline 1 & 0.937 & 0.000 & 1 & 320 & 30 & 15 \\
2 & 0.507 & 2.980 & 4 & 152 & 30 & 15 \\
3 & 0.526 & 3.565 & 4 & 224 & 40 & 15 \\
4 & 0.516 & -5.078 & 4 & 37 & 40 & 15 \\
5 & 0.443 & 2.195 & 4 & 220 & 40 & 15 \\
6 & 0.500 & 0.054 & 4 & 176 & 40 & 15 \\
\hline
\end{tabular}

* Error is not cumulative since it is arrested by each FEA
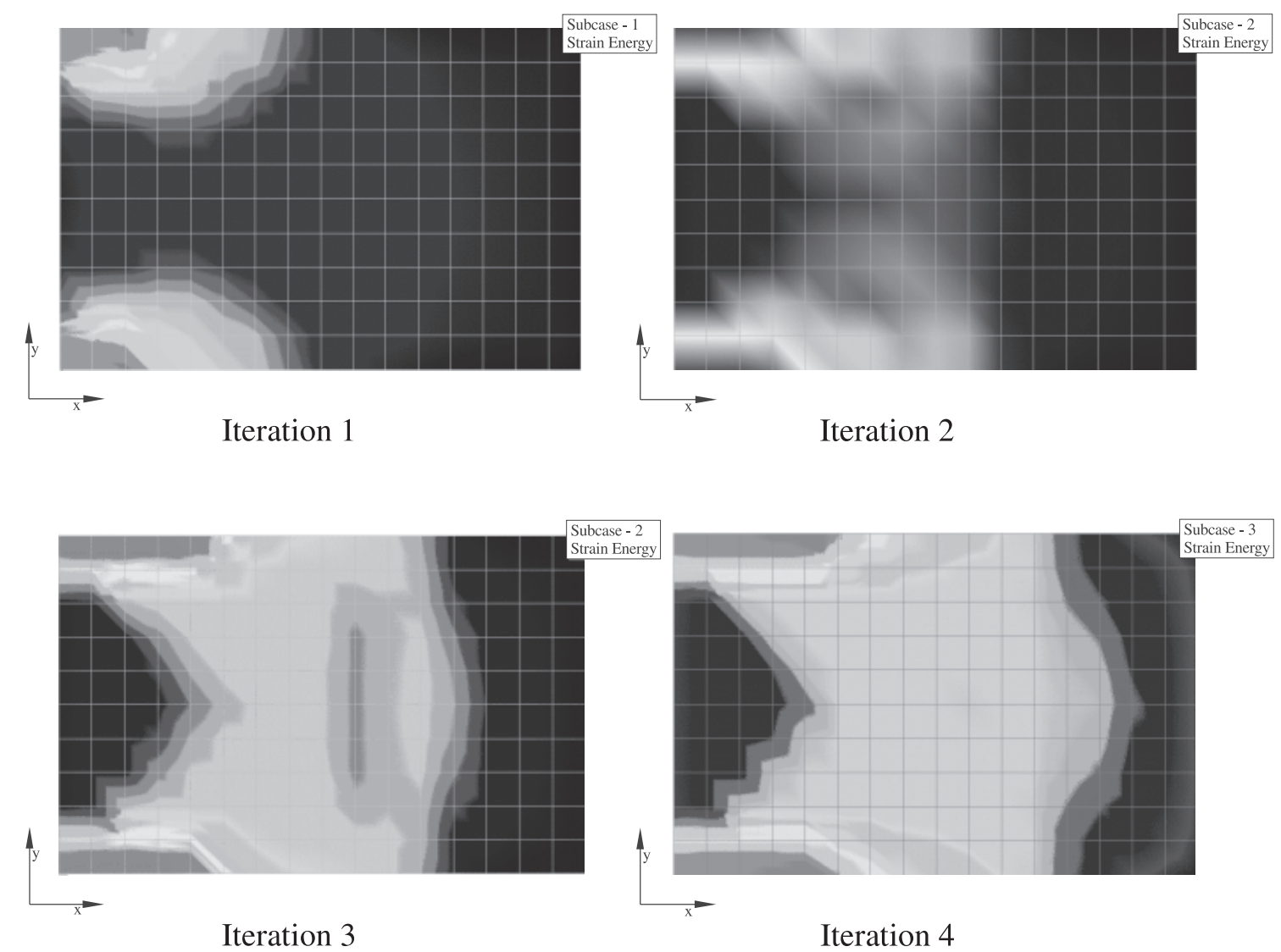

Fig. 4 Modal dynamic strain energy distribution after dynamic redesign (160 solid elements cantilever plate); $\omega_{2}^{\prime 2} / \omega_{2}^{2}=10.895$; strain energy normalized in the range $0-1$

Table 8 Results of the 160 elements model at each iteration for modal dynamic redesign objective

\begin{tabular}{|c|c|c|c|c|c|c|}
\hline \multirow{2}{*}{$\begin{array}{l}\text { Iter } \\
\#\end{array}$} & \multicolumn{2}{|c|}{ Dynamic redesign } & \multirow{2}{*}{$\begin{array}{l}\text { \# of } \\
\text { increments }\end{array}$} & \multirow{2}{*}{$\begin{array}{l}\text { \# of redesign } \\
\text { variables }\end{array}$} & \multirow{2}{*}{$\begin{array}{l}\# \text { of } \\
\text { modes }\end{array}$} & \multirow{2}{*}{$\begin{array}{l}\text { Increment } \\
\text { size }(\%)\end{array}$} \\
\hline & $\omega_{2}^{\prime 2} / \omega_{2}^{2}$ & Error $(\%)^{*}$ & & & & \\
\hline 1 & 1.871 & 0.778 & 5 & 80 & 30 & 15 \\
\hline 2 & 1.845 & 1.702 & 5 & 20 & 30 & 15 \\
\hline 3 & 1.696 & 2.534 & 4 & 40 & 30 & 15 \\
\hline 4 & 1.861 & 1.928 & 5 & 54 & 30 & 15 \\
\hline
\end{tabular}

* Error is not cumulative since it is arrested by each FEA 
Table 9 Results of the 160 elements model (1122 d.o.f.'s) at each iteration for static and dynamic redesign objectives

\begin{tabular}{|c|c|c|c|c|c|c|c|c|}
\hline \multirow{2}{*}{$\begin{array}{l}\text { Iter } \\
\#\end{array}$} & \multicolumn{2}{|c|}{ Static redesign } & \multicolumn{2}{|c|}{ Dynamic redesign } & \multirow{2}{*}{$\begin{array}{l}\text { \# of } \\
\text { increments }\end{array}$} & \multirow{2}{*}{$\begin{array}{l}\text { \# of redesign } \\
\text { variables }\end{array}$} & \multirow{2}{*}{$\begin{array}{l}\# \text { of } \\
\text { modes }\end{array}$} & \multirow{2}{*}{$\begin{array}{l}\text { Increment } \\
\text { size }(\%)\end{array}$} \\
\hline & $u_{289}^{\prime} / u_{289}$ & Error $(\%)^{*}$ & $\omega_{2}^{\prime 2} / \omega_{2}^{2}$ & Error $(\%)^{*}$ & & & & \\
\hline 1 & 0.492 & 2.813 & 1.871 & 0.380 & 5 & 160 & 30 & 15 \\
\hline 2 & 0.494 & 6.840 & 1.831 & 1.911 & 5 & 142 & 30 & 15 \\
\hline 3 & 0.535 & -1.462 & 1.706 & -0.259 & 4 & 132 & 30 & 15 \\
\hline 4 & 0.508 & 4.721 & 1.783 & 2.564 & 5 & 134 & 30 & 15 \\
\hline
\end{tabular}

* Error is not cumulative since it is arrested by each FEA
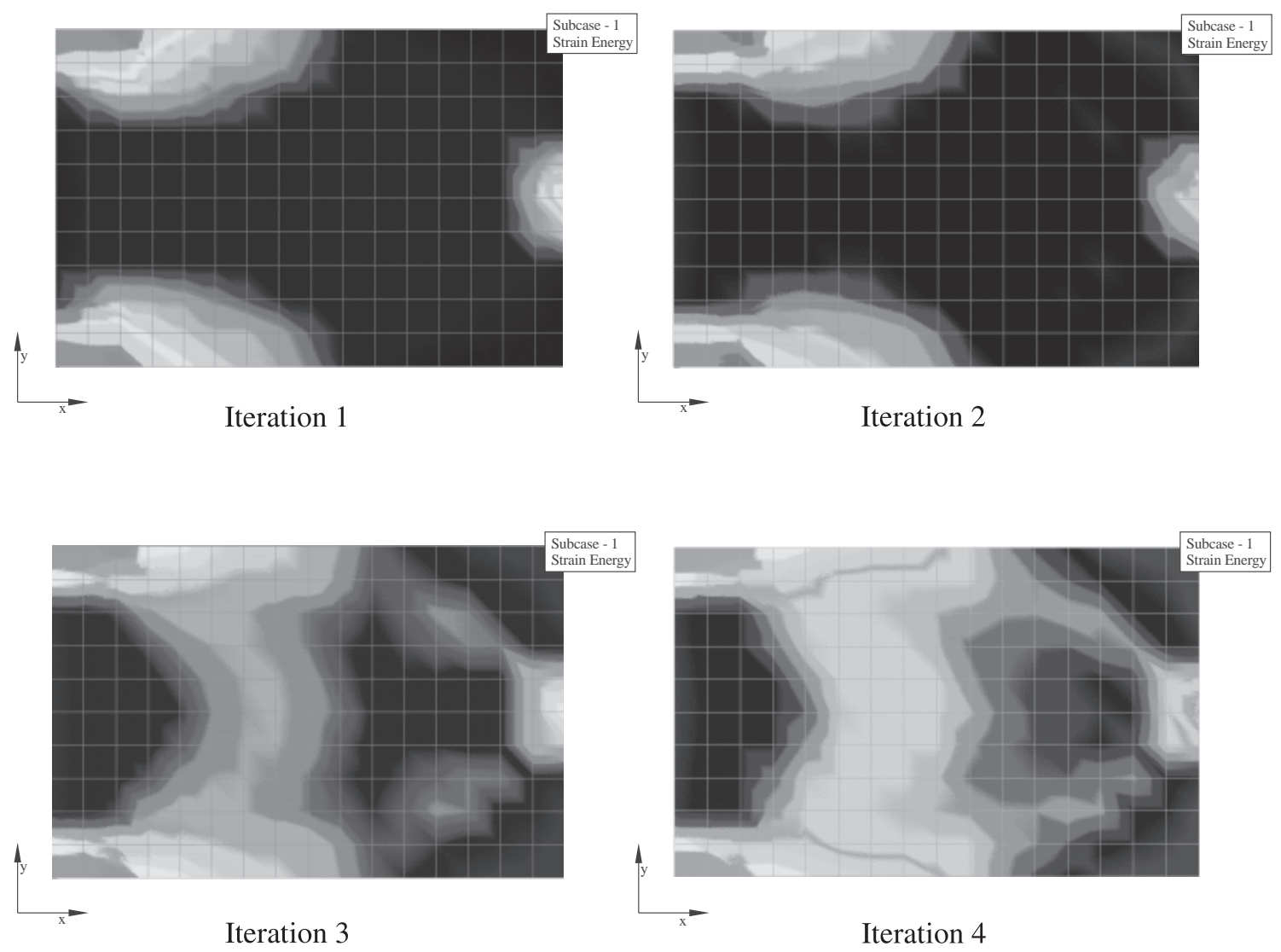

Fig. 5 Static/modal dynamic strain energy distribution after static/dynamic redesign (160 solid elements cantilever plate); $\omega_{2}^{\prime 2} / \omega_{2}^{2}=10.421 ; u_{289}^{\prime} / u_{289}=0.0661 ;$ strain energy normalized in the range $0-1$

The cross bracing from the modal dynamic redesign has more openings in it and is distinctively different, as shown in Figs. 2 and 3. Near the free end, the influence of static redesign is apparent with the evolution of the Gothic arc structure. The modal dynamic strain energy density shows very low strain energy density near the free end. Thus, static strain energy density dominates the redesign evolution.

\section{5}

\section{Conclusions}

A LargE Admissible Perturbations (LEAP) methodology has been developed to perform integrated topology and structural performance redesign. The topological redesign evolves from a solid block using the modulus of elasticity of elements as redesign variables with a minimum change optimality criterion. Strain energy density levels are used to freeze low energy elements from the redesign process in a specific iteration. Elements frozen in a specific iteration may be reactivated in subsequent iterations. At the end, elements of no consequence may be removed. Compared to other topology redesign methods, the method developed in this paper makes it possible to work in continuous design space instead of "binary" design space. Thus, adequate resolution is achieved using far fewer elements.

Structural performance specifications may be imposed by the designer on static deflections and for modal 
dynamic properties. The developed LEAP methodology and solution algorithm can perform integrated topology and structural performance redesign with satisfactory accuracy in a single iteration (no FEA's) for performance changes on the order of $100 \%-300 \%$ without trial and error. For larger changes, on the order of $3300 \%$, four to six iterations are used to achieve the same level of accuracy. One FEA is used per iteration without trial and error. The benchmarking problem of redesigning a solid plate cantilever has been solved for integrated topology and performance with the addition of static deflection and/or natural frequency constraints. Numerical results show the accuracy and efficiency of the LEAP algorithm particularly after introduction of the static mode compensation in the static deflection general perturbation equations. Use of two finite element models with different resolution - 1122 d.o.f.'s and 4158 d.o.f.'s-produce similar topologies indicating uniqueness of solution. Structural performance constraints have a strong effect on the final topology producing significantly different solution. Prevailing however, in the redesigned topology is the feature of gothic arc bracing. The latter is different from the straight truss bracings observed in many solutions of the benchmarking problems published in the literature.

All redesign sequences shown in Figs. 2-5 show the structural evolution during the redesign process. The final shape in each figure indicates where stiffening is required. From the results of the redesign process, we get also the values of element property changes and therefore physical modifications can be done accordingly. Stiffening is proportional to the calculated energy density.

Acknowledgements This work was funded partially by the Ship Structures and Systems Division of the Office of Naval Research, Contract \#1 DOD-G-N00014-96-1-0909.

\section{References}

Alzahabi, B.; Bernitsas, M.M. 1992: Cylindrical shell redesign by large admissible perturbations. In: Proc. ASCE Engineering Mechanics Conf. (held in College Station, TX) pp. 200-203

Bendsøe, M.P.; Kikuchi, N. 1988: Generating optimal topologies in structural design using a homogenization method. Comp. Meth. Appl. Mech. Engrg. 71, 197-224

Bernitsas, M.M. (ed.) 1994: RESTRUCT: Vol. I-Theoretical manual, Vol. II-User's manual, Vol. III-Application manual. Report to the University of Michigan/Sea Grant/Industry Consortium in Offshore Engineering, Department of Naval Architecture and Marine Engineering. Ann Arbor: University of Michigan

Bernitsas, M.M.; Kang, B. 1991: Admissible large perturbation in structural redesign. AIAA J. 29, 104-113
Bernitsas, M.M.; Rim, C.W. 1994: Redesign of plates by large admissible perturbations. AIA A J. 32, 1021-1028

Bernitsas, M.M.; Suryatama, D. 1999: Structural redesign by large admissible perturbations with static mode compensation. J. Offshore Mech. Arctic Engrg., Trans. ASME 121, $39-46$

Bernitsas, M.M.; Tawekal, R.L. 1991: Structural model correlation using large admissible perturbation in cognate space. AIAA J. 29, 2222-2232

Bernitsas, M.M.; Suryatama, D.; Kang, B.; Karr, D.G. 1994: Shape and topology structural redesign by large admissible perturbations. Proc. Solid Freeform Fabrication Symp. (held in Austin, TX), pp. 285-292

Beyko, E.; Bernitsas, M.M. 1993: Reliability of large scale structures by large admissible perturbations. J. Offshore Mech. Arctic Engrg., Trans. ASME 115, 167-178

Bremicker, M.; Chirehdast, M.; Kikuchi, N.; Papalambros, P.Y. 1991: Integrated topology and shape optimization in structural design. Mech. Struct. Mach. 19, 551-587

Choi, K.K.; Haug, E.J.; Seong, H.G. 1983: An iterative method for finite dimensional structural optimization problems with repeated eigenvalues. Int. J. Numer. Meth. Engrg. 19, 93-112

Gill, P.E.; Murray, W.; Saunders, M.A.; Wright, M.H. 1983a: User's guide for SOL/QPSOL: A FORTRAN package for quadratic programming. Department of Operation Research, Stanford University

Gill, P.E.; Murray, W.; Saunders, M.A.; Wright, M.H. 1983b: User's guide for SOL/NPSOL: A FORTRAN package for nonlinear programming. Department of Operation Research, Stanford University

Haber, R.B.; Jog, C.S.; Bendsøe, M.P. 1995: The perimeter method - A new approach to variable-topology shape design. In: Olhoff, N.; Rozvany, G.I.N. (eds.) Proc. First World Congress of Structural and Multidisplinary Optimization, WCSMO-1 (held in Goslar, Germany), pp. 153-160. Oxford: Pergamon

Haug, E.J.; Choi, K.K.; Komkov, V. 1986: Design sensitivity analysis of structural systems. New York: Academic Press

Hoff, C.J.; Bernitsas, M.M. 1985: Dynamic redesign of marine structures. J. Ship Res. 29, 285-295

Hoff, C.J.; Bernitsas, M.M. 1986: Static redesign of offshore structures. In: Proc. 5-th Int. OMAE Symp. (held in Tokyo), Vol. III, pp. 78-85

Kang, B.; Bernitsas, M.M. 1994: Stress redesign by large admissible perturbations. Proc. BOSS '94 Conf. (held in Cambridge, MA), Vol. 3, pp. 201-212

Kang, B.; Beyko, E.; Bernitsas, M.M. 1992: Invariant and consistent redundancy by large admissible perturbations. Marine Struct. 5, 23-70

Kim, J.H.; Bernitsas, M.M. 1990: Redesign of marine structures. Marine Struct. 1, 139-183

Lipton, R.; Diaz, A. 1995: Moment formulations for optimum layout in 3D elasticity. In: Olhoff, N.; Rozvany, G.I.N. (eds.) Proc. First World Congress of Structural and Multidisplinary Optimization, WCSMO-1 (held in Goslar, Germany), pp. 161-168. Oxford: Pergamon

Sandstrom, R.E.; Anderson, W.J. 1982: Modal perturbation methods for marine structures. Trans., SNAME 90, 41-54 
Schittkowski, K. 1986: QLD: A FORTRAN code for quadratic programming, user's guide. Mathematisches Institut, Universität Bayreuth, Germany

Stetson, K.A. 1975: Perturbation method of structural design relevant to holographic vibration analysis. AIAA J. 13, 457-459

Stetson, K.A.; Harrison, I.R. 1981: Redesign of structural vibration modes by finite element inverse perturbation. ASME Trans., J. Engrg. Power 103, 319-325

Tawekal, R.L.; Bernitsas, M.M. 1992: Finite element model correlation for offshore structures. J. Offshore Mech. Arctic Engrg., ASME Trans. 114, 154-164

Tits, A.L.; Zhou, J.L. 1993: User's guide for FSQP Version 3.3b: A FORTRAN code for solving constrained nonlinear (minimax) optimization problems, generating iterates satisfying all inequality and linear constraints. Electrical Engineering Department and Institute for Systems Research, Univ. of Maryland, College Park, MD

Yang, R.J.; Chuang, C.H. 1992: Optimal topology design using linear programming. Research Report, CAE Department, Ford Motor Company

\section{6}

\section{Appendix A: General perturbation equation for modal dynamics}

The counterpart of (2) for the objective structure in State $\mathrm{S} 2$ is

$\mathbf{K}^{\prime}=\mathbf{M}^{\prime} \boldsymbol{\omega}^{\prime 2}$,

where the primed quantities refer to State $\mathrm{S} 2 . \mathbf{K}^{\prime}$ is the generalized stiffness matrix of the objective structure

$\mathbf{K}^{\prime}=\boldsymbol{\Phi}^{\prime T} \mathbf{k}^{\prime} \boldsymbol{\Phi}^{\prime}$

and $\mathbf{M}^{\prime}$ is the generalized mass matrix of the objective structure

$\mathbf{M}^{\prime}=\boldsymbol{\Phi}^{\prime T} \mathbf{m}^{\prime} \boldsymbol{\Phi}^{\prime}$

Substituting relationships (5)-(8) and (11)-(12) into (26) the dynamic general perturbation equation is developed as

$\Phi^{\prime T} \Delta \mathbf{k} \boldsymbol{\Phi}^{\prime}-\boldsymbol{\Phi}^{\prime T} \Delta \mathbf{m} \boldsymbol{\Phi}^{\prime} \boldsymbol{\omega}^{\prime 2}=\boldsymbol{\Phi}^{\prime T} \mathbf{m} \boldsymbol{\Phi}^{\prime} \boldsymbol{\omega}^{\prime 2}-\boldsymbol{\Phi}^{\prime T} \mathbf{k} \boldsymbol{\Phi}^{\prime}$.

(29) consists of $n^{2}$ scalar equations of the following form:

$\sum_{e=1}^{p}\left(\boldsymbol{\Psi}_{j}^{\prime T} \mathbf{k}_{e} \boldsymbol{\Psi}_{i}^{\prime}-\omega_{i}^{\prime 2} \boldsymbol{\Psi}_{j}^{\prime T} \mathbf{m}_{e} \boldsymbol{\Psi}_{i}^{\prime}\right) \alpha_{e}=$

$\omega_{i}^{\prime 2} \boldsymbol{\Psi}_{j}^{\prime T} \mathbf{m} \boldsymbol{\Psi}_{i}^{\prime}-\boldsymbol{\Psi}_{j}^{\prime T} \mathbf{k} \boldsymbol{\Psi}_{i}^{\prime}, \quad i, j=1,2, \ldots, n$
7

Appendix B: General perturbation equation for static deflection

The derivation of the general perturbation equation for static deflection without static mode compensation is presented in this appendix. Development of this general perturbation equation using static mode compensation can be seen in the paper by Bernitsas and Suryatama (1999).

The counterpart of (4) can be written as

$\mathbf{k}^{\prime} \mathbf{u}^{\prime}=\mathbf{f}$,

where it is assumed that $\mathbf{f}^{\prime}=\mathbf{f}$. For $f^{\prime} \neq \mathbf{f}$, see Tawekal and Bernitsas (1992). Let $\mathbf{Q}^{\prime}$ be the transformed displacement vector which is defined - without static mode compensation - as

$\mathbf{u}^{\prime}=\Phi^{\prime} \mathbf{Q}^{\prime}$.

Substituting (32) into (31) gives

$\mathbf{k}^{\prime} \boldsymbol{\Phi}^{\prime} \mathbf{Q}^{\prime}=\mathbf{f}$.

Premultiplying (33) by $\boldsymbol{\Phi}^{\prime T}$ yields

$\boldsymbol{\Phi}^{\prime T} \mathbf{k}^{\prime} \boldsymbol{\Phi}^{\prime} \mathbf{Q}^{\prime}=\boldsymbol{\Phi}^{\prime T} \mathbf{f}$

Since $\mathbf{K}^{\prime}=\boldsymbol{\Phi}^{\prime T} \mathbf{k}^{\prime} \boldsymbol{\Phi}^{\prime}$, (34) becomes

$\mathbf{K}^{\prime} \mathbf{Q}^{\prime}=\boldsymbol{\Phi}^{\prime T} \mathbf{f}$,

or

$\mathbf{Q}^{\prime}=-\frac{1}{\mathbf{K}^{\prime}} \boldsymbol{\Phi}^{\prime T} \mathbf{f}$.

Applying (36) to (32) gives

$\mathbf{u}^{\prime}=\boldsymbol{\Phi}^{\prime} \frac{1}{\mathbf{K}^{\prime}} \boldsymbol{\Phi}^{\prime T} \mathbf{f}$.

Then, the components of vector $\mathbf{u}^{\prime}$ can be obtained as

$u_{i}^{\prime}=\sum_{m=1}^{n_{r}} \frac{\Psi_{i m}^{\prime} A_{m}}{B_{m}+\sum_{e=1}^{p} C_{m e} \alpha_{e}}$,

where

$A_{m}=\sum_{j=1}^{n_{r}} \Psi_{j m}^{\prime} f_{j}$,

$B_{m}=\boldsymbol{\Psi}_{m}^{\prime}{ }^{T} \mathbf{k}_{e} \boldsymbol{\Psi}_{m}^{\prime}$,

$C_{m e}=\boldsymbol{\Psi}_{m}^{\prime T} \mathbf{k}_{e} \boldsymbol{\Psi}_{m}^{\prime}$,

and $\Psi_{j m}^{\prime}$ represents the amplitude of the $j$-th d.o.f. of mode $m$. 


\section{8}

\section{Nomenclature}

$\mathbf{B}_{e} \quad$ element strain-nodal displacement matrix

$\mathbf{D}_{e} \quad$ element constitutive law matrix

d.o.f.('s) degree(s) of freedom

$\mathrm{E} \quad$ Young's modulus

$E_{e} \quad$ element Young's modulus

FSQP Feasible Sequential Quadratic Programming solver

$f_{i} \quad$ natural frequency of the $i$-th mode

$\mathbf{k}, \mathbf{K}$ global and generalized stiffness matrix

$\mathbf{k}_{e} \quad$ stiffness matrix of element or group of elements related to property $e$

LEAP LargE Admissible Perturbation

$\mathbf{m}, \mathbf{M}$ global and generalized mass matrix

$\mathbf{m}_{e}$ mass matrix of element or group of elements related to property $e$

$n \quad$ number of degrees of freedom of structural model

$n_{a} \quad$ number of admissibility equations used in redesign

$n_{e} \quad$ number of elements of structural model

$\mathbf{N}_{e} \quad$ interpolation function matrix for each element

NPSOL Nonlinear Programming SOLver

$n_{r} \quad$ number of the extracted modal dynamic modes

$n_{u} \quad$ number of displacement constraints

$n_{\omega} \quad$ number natural frequency constraints

$p \quad$ number of redesign variables

PAR Perturbation approach to redesign
$\mathbf{Q}^{\prime} \quad$ transformed displacement vector

QPSOL Quadratic Programming SOLver

RESTRUCT program for Redesign of STRUCTures

S1 the baseline state of the structure to be designed; the structural performance is known but does satisfy designer specifications

S2 the unknown objective structural state; structure should have satisfied designer specifications after completing this state

u nodal static displacement vector

$\mathbf{u}_{b}$ nodal static displacement vector of the baseline structure or the structure at the previous increment

$V_{e} \quad$ the element volume

\section{1}

\section{Greek symbols}

$\alpha_{e} \quad$ fractional change of an element or group of element

$\Delta \quad$ changes between the initial State S1 and the unknown State S2

$\Phi$ dynamic mode shape matrix

$\boldsymbol{\Psi}_{i} \quad i$-th dynamic mode shape vector

$\omega_{i}^{2} \quad i$-th modal dynamic eigenvalue

$\rho_{e} \quad$ element density 